\title{
Erratum: Feeder-independent culture of human embryonic stem cells
}

Tenneille E Ludwig, Veit Bergendahl, Mark E Levenstein, Junying Yu, Mitchell D Probasco \& James A Thomson

Nat. Methods 3, 637-646 (2006); published online 21 July 2006; corrected after print 24 August 2006.

In the version of this Protocol initially published, the references were numbered incorrectly. This error has been corrected in the HTML and PDF versions of the article. 